\title{
Primeras indagaciones sobre derecho a la ciudad y espacios de vida de las personas trans en Mar del Plata ${ }^{1}$
}

Federico García Fernández ${ }^{2}$

Universidad Nacional de Mar del Plata

\section{Artículo científico}

Material original autorizado para su primera publicación en el Journal de Ciencias Sociales, Revista Académica de la Facultad de Ciencias Sociales de la Universidad de Palermo.

Recepción: 16-02-2021

Aceptación: 5-10-2021

Resumen: Las ciudades se encuentran compuestas por tantas realidades como personas que las habitan. Sin embargo, es en las últimas décadas que desde la academia se ha comenzado a dar lugar a las disidencias en los estudios espaciales. La incorporación de la voz de la población, desde la Geografía sensible, suma a los patrones de movilidad la comprensión de las prácticas espaciales y de los sentidos que los habitantes asignan al territorio. El presente trabajo es una primera aproximación a las comunidades trans de la ciudad de Mar del Plata (Buenos Aires, Argentina) desde los sustentos de la Geografía de Género. Se propone como objetivo, identificar y comprender los espacios vividos por dicha población a través de sus prácticas espaciales y de los sentidos asignados al territorio. El trabajo de campo se ha desarrollado en dos momentos metodológicos de obtención de información de primera mano. Inicialmente, se han aplicado conversaciones con contenido, las cuales han permitido un primer acercamiento y reconocimiento de las diferentes realidades. Luego, se decidió utilizar cuestionarios virtuales, con el objetivo de acceder a la población y comprender las realidades con las que las personas conviven a diario en la ciudad. La intención de lo realizado fue reconstruir territorios cotidianos a partir del reconocimiento de la dinámica de los espacios de frecuentación de población trans, para aportar conocimiento a esta línea de investigación dentro del campo geográfico latinoamericano.

\footnotetext{
${ }^{1}$ Este trabajo comenzó a delinearse en el transcurso de la cátedra "Problemáticas territoriales: ¿Como analizamos su estudio y comprensión?" A cargo de la Dra. Patricia Lucero, la Dra. Claudia Mikkelsen, la Dra. Sofía Ares y el Dr. Fernando Sabuda, a quienes reconozco y admiro. Luego se enriqueció con las lecturas recurrentes de Claudia Mikkelsen y Agustín Parracone, a quienes agradezco enormemente sus comentarios, debates y acompañamiento.

2 Estudiante avanzado de la carrera Licenciatura en Geografía en la Universidad Nacional de Mar del Plata (Argentina). Trabaja en temas que abocan a la Geografía Posmoderna Feminista, haciendo foco en temáticas de género y bienestar en las poblaciones LGBTIQ+.

Correo electrónico: fedegarciafernandez99@gmail.com
}

pág. 28 
Palabras claves: Geografía de género; espacios de vida; Queer; acceso a la ciudad.

\title{
First inquiries about the right to the city and living spaces of trans people in Mar del Plata
}

\begin{abstract}
Cities are made up of as many realities as there are people who inhabit them. However, it's in recent decades that the academy has begun to give rise to dissidence in spatial studies. The incorporation of the voice of the subjects, from sensitive geography, adds to the mobility patterns, the understanding of spatial practices and the meanings that the inhabitants assign to the territory. The present work's a first approach to the trans communities of the city of Mar del Plata (Buenos Aires, Argentina) from the Geography of Gender. The objective's to identify and understand the spaces lived by trans subjects through their spatial practices and the meanings assigned to the territory. To develop this work, the methodology has been divided into two moments of obtaining first-hand information. Initially, conversations with content have been held, which have allowed a first approach and recognition of the different realities. Then, it was decided to use virtual questionnaires, with the aim of accessing the population and understanding the realities with which they live daily in the city. With this, it was sought to reconstruct everyday territories from the recognition of the dynamics of the frequentation spaces of trans subjects, to contribute knowledge to this line of research within the Latin American geographic field.
\end{abstract}

Keywords: Geography of gender; space of living; Queer; right to the city.

\section{Introducción}

Al fin y al cabo somos lo que hacemos para cambiar lo que somos. La identidad no es una pieza de museo, quietecita en la vitrina, sino la siempre asombrosa síntesis de las contradicciones nuestras de cada día.

Galeano, El libro de los Abrazos (1989).

La forma en que las personas ${ }^{3}$ habitan y se apropian de los espacios ha sido una temática muy nutrida en las últimas décadas dentro de la disciplina geográfica. Los estudios de género lentamente cobran auge y buscan instalarse con fuerza dentro de las agendas latinoamericanas. La combinación de ambas permite abrir puertas a nuevos interrogantes, a

\footnotetext{
${ }^{3} \mathrm{Si}$ bien se ha realizado un esfuerzo en intentar desarrollar este trabajo en un lenguaje neutro, cuando se utilicen expresiones que tiendan a recaer en el binomio varón-mujer (a/o o as/os), dada la diversidad genérica que se busca

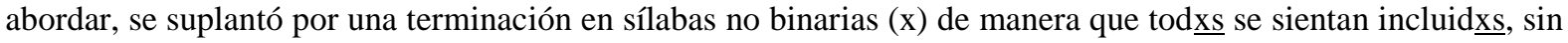
complejizar por de más la lectura.
} 
nuevas formas de estudiar, conocer y comprender los diferentes lugares que conforman los espacios urbanos.

La Geografía del Género en Latinoamérica se presenta abocada al estudio de las prácticas sociales de producción y reproducción del espacio que han sido invisibilizadas diferencialmente entre las relaciones de poder más elementales y que han gestado territorialidades injustas, "el espacio se manifiesta como un instrumento de control social y de discriminación que respalda la dominación masculina en la sociedad" (Lan, 2016, p. 55). Surgen así, como motor de cambio, para proponer nuevas espacialidades que permitan la visibilidad de las diferencias dentro de las complejas relaciones que acontecen en el espacio.

La ciudad de Mar del Plata es una localidad costera, cabecera del partido de General Pueyrredon, localizada al Sudeste de la provincia de Buenos Aires, Argentina (Figura 1). Con un valor proyectado para 2020 de 656.456 habitantes según el Instituto Nacional de Estadísticas y Censos (INDEC, 2020). Se ubica en el séptimo puesto en relación a la cantidad de habitantes del país, y es catalogada por el mismo organismo dentro de las Aglomeraciones de Tamaño Intermedio Mayor (ATIs).

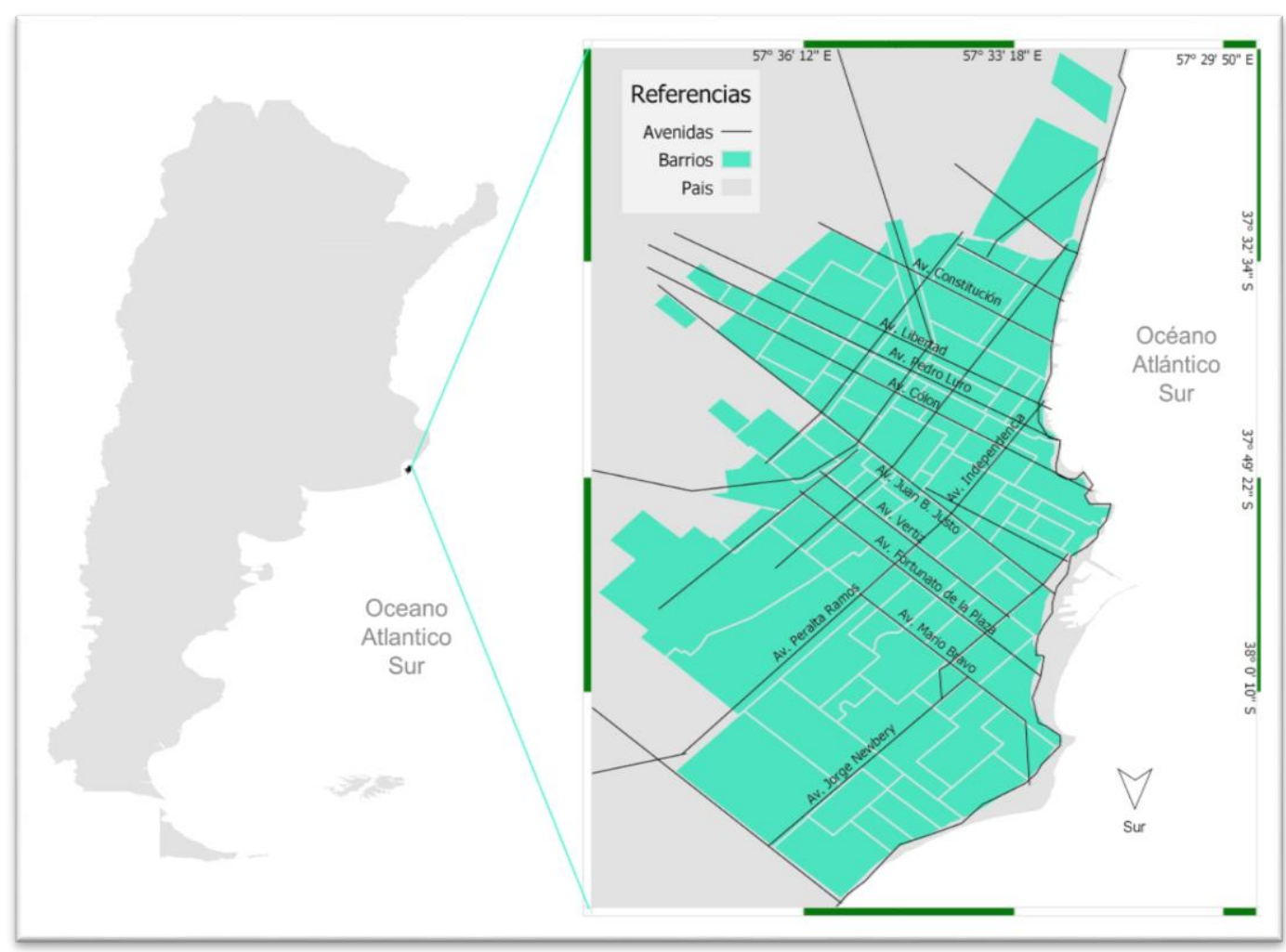

Figura 1: Localización de la ciudad de Mar del Plata según barrios

Fuente: Elaboración personal mediante el empleo del SIG de acceso libre Qgis. 
Partiendo de la necesidad de dar voz a las disidencias dentro de las investigaciones, actuando como interlocutores respetuosos y empáticos, brindando un espacio de expresión y, procurando así, comprender las variadas realidades que se viven en la ciudad, este artículo busca hacer un primer reconocimiento de la dinámica urbana y los espacios de vida de la población trans en Mar del Plata para el año 2019-2020, en pos de evidenciar las posibilidades de acceso a la ciudad. Se analizaron territorios cotidianos a partir de una reconstrucción en base a espacios de frecuentación, concibiéndolos como lugares en los que las personas establecen lazos y construyen parte de sus territorios cotidianos (Bailly, 1989), para aportar conocimiento a esta línea de investigación dentro del campo geográfico latinoamericano y brindar información al activismo local.

Esta propuesta se enmarca dentro de las llamadas "Geografías Sensibles" (Hiernaux, 2008), un método de estudio que permite rescatar la dimensión emotiva de la experiencia espacial, para reconstruir los imaginarios sociales y las relaciones de Ixs sujetxs que habitan el espacio. Se debe aspirar, como investigadores, a dejar de condicionar social y culturalmente, para pasar a ser un instrumento que permita cuestionar el conocimiento real, universal y neutro, haciendo foco en el lugar, en la horizontalidad y en la deconstrucción como bandera de empoderamiento de la población (Ibarra-García y Escomilla-Herrera, 2016). Buscamos examinar los cambios en los lugares que habitamos, con base en las relaciones sociales, las cuales se encuentran transversalizadas por las relaciones de género como fenómeno que afecta, no sólo los procesos, sino también las manifestaciones en el espacio (García-Ramón, 2008).

La metodología que se ha decidido aplicar se compone de dos momentos. Un inicio de naturaleza cualitativa, en línea con lo propuesto por Scribano (2008), para obtener datos primarios sobre el universo de estudio, por medio de conversaciones con contenido que cumplieran la función de un primer acercamiento. Dichas entradas a campo fueron realizadas en el mes de noviembre del año 2019, con una participación de cuatro sujetxs. En un segundo momento se realizó un abordaje cuantitativo (Sautu et al. 2005, p. 52), para trabajos que son de naturaleza "microsocial" focalizando en las experiencias individuales y las interacciones sociales como fuentes de creación de significados. Así, se aplicaron cuestionarios relevados por medio de Google Forms, entre los meses de mayo y junio del año 2020, reuniendo un total de 20 casos localizados en 15 barrios de la ciudad de Mar del Plata. El objetivo era relevar cuatro dimensiones: Espacios de vida (Bailly, 1989), movilidad cotidiana (Ares, 2012), espacios topofílicos y topofóbicos (Tuan, 2007) y acceso a la ciudad (Harvey, 2012).

A continuación, el trabajo se estructurará en cuatro secciones. Para iniciar, se debatirán los conceptos troncales: acceso a la ciudad, espacio vivido, movilidad cotidiana, espacios del miedo, topofilia y topofobia; y la noción del espacio urbano como construcción 
social. Acto seguido, se presentarán en profundidad los materiales y los métodos adoptados. Sin más, se culmina con la presentación de los resultados obtenidos, que fueron analizados a la luz de la perspectiva teórica. Una breve reflexión cumple la función de cierre de esta primera indagación.

\section{Estado del arte}

El presente trabajo se enmarca regionalmente en un contexto de violencia hacia la comunidad trans. Situación que queda plasmada en los 2.343 homicidios por transodio ocurridos entre 2008 y 2017, de los cuales 2.048 sucedieron en América del Sur y Central, en una población cuya esperanza de vida no supera los 40 años de edad, y en la que el $57 \%$ de las personas disidentes no superan los 29 años de edad (Vila Núñez, 2018). Por esta coyuntura, y tratándose de un estudio de tipo local, se han priorizado materiales que provengan del interior de la región latinoamericana, sin desconocer otras líneas vigentes de investigación europeas, africanas o anglosajonas.

El surgimiento de una nueva ola feminista latinoamericana, en Argentina y diferentes países en las últimas dos décadas, ha funcionado como herramienta para abrir el diálogo feminista a la Geografía desde una posición posmoderna, que lentamente va cobrando fuerza en tareas de visibilización y abordaje de problemáticas de género, teoría queer y otras categorías de sexualidad (Lan y Veleda Da Silva, 2007).

En la actualidad, de forma naciente y muy acotada, Lan (2016) y Colombara (2017; 2019), reconocen temáticas de investigación dentro de la Geografía de género y feminista en Latinoamérica y Argentina. Las principales están relacionadas con: los movimientos de mujeres y el feminismo; las relaciones género y la utilización diferencial del espacio; los femicidios y la violencia hacia la mujer; la explotación y la trata sexual; la prostitución; la diversidad e identidad sexual; entre otras.

En cuanto a las líneas de investigación que abordan específicamente a la población trans, se encuentran mucho más dispersas y focalizadas, pudiendo recuperar, por ejemplo, a Torres-Rodríguez (2012) y Fuentealba-Matus (2016) desde Chile; en Argentina a Butierrez (2019) y Larreche y Ercolani (2018); y con más fuerza, desde Brasil, a Silva et al. (2013) o a Barbosa de Souza y Ornat (2019).

En cuanto a la Geografía marplatense, no se han encontrado trabajos que aborden Geografías de las sexualidades. Sin embargo, desde el campo disciplinar de la Sociología se reconoce la línea de investigación de Darouiche (2019), con un interesante aporte sobre mujeres trans-travestis que ejercen sexo comercial en diferentes espacios públicos de la ciudad de Mar del Plata. 


\section{Perspectiva teórica}

Las ciudades, como la compleja yuxtaposición de sistemas de vida, íntegramente cambiantes y dinámicos, aglomeran por excelencia la suma de las actividades sociales, productivas y materiales. Desde un funcionamiento condicionado por las cuestiones sociales, políticas y económicas, orientan el quehacer de Ixs individuos, considerándolos actores activos y productores del espacio, con base en sus propios valores y aprendizajes. A esta suma la concebiremos como dinámica urbana (Jiménez-Jiménez et al. 2014).

La identificación con el territorio, la percepción personal, Ixs individuxs como interlocutores válidos para la imbricación sociedad-espacio, a través de los caracteres simbólicos de los mitos espaciales, de visiones funcionalistas o visiones parcializadas, permiten llegar a reconstruir el estudio de los espacios de vida de diferentes personas y sus lazos tanto simbólicos como lineales con los lugares. Las escuelas, las iglesias, los edificios, las plazas... cada comunidad construye, en base a su espacio, una estructura de localización y marginación que se podrá estudiar y evidenciar en base a la pluralidad de mundos imaginarios que confluyen en la construcción urbana (Bailly, 1989).

La ciudad es percibida y a la vez representada por las poblaciones que viven en ella. Así se constituye en su interior un mosaico de lugares con diferente accesibilidad psicológica, formando barrios concebidos como el terreno conocido de dominio público de esas poblaciones. Fuera de estos, la capacidad de conocer responderá a las propias experiencias, ya que, todas las características que construyen a la persona influyen en su imagen del territorio, en los lugares, y en su identidad. Podemos llegar a considerar a la ciudad como conjuntos de grupos que actúan entre sí y con el medio, generando lazos complejos, influyendo en las decisiones del individuo y de estx con el entorno (Bailly, 1979).

Esta construcción del espacio con peso en la percepción individual y grupal, nos da como resultado distintos lugares, que pueden ser clasificados, siguiendo a Tuan (2007), como topofílicos, es decir, aquellos en los que las personas establecen lazos afectivos con ese ambiente circundante, el cual responde a una experiencia personal. Son los "vínculos afectivos del ser humano con su entorno material" (Tuan, 2007, p. 130) los cuales se producen por las sensaciones que generan en la persona, así como por la internalización de la historia del lugar. Por el contrario, también encontraremos las relaciones topofóbicas, que aluden a conexiones negativas entre el sujeto y el espacio. Poseer desconfianza o miedo de lugares, automáticamente genera estrategias de evasión y restricción en su circulación, buscando oportunidades alternativas para resolver el mismo fin. 
Estos, deben ser complementados, suma Lindón (2009, p. 6), con "el estudio del cuerpo y las emociones como ventana para comprender la construcción social de la ciudad, de lo urbano, de sus lugares, a través de los sujetos que la habitan corporal y emocionalmente". Incorporar también la construcción social de espacios de miedo, terror y violencia -ya sea por los sucesos socio históricos, o por la carga social que se les adjudicagenera que este se cargue de emociones, quedando en la memoria la impronta de lo que haya sucedido.

Los lugares no pueden ser concebidos como inmutables ni imparciales en el proceso de construcción social de la ciudad. Cada persona habita (Bailly, 1979) y se acerca a los lugares que cobran interés según su mirada (Lindón, 2009) produciendo el espacio y creando vínculos con el mismo. "Todas las prácticas espaciales que despliega el sujeto cuerpo están teñidas de sentimientos, afectos y generan en los sujetos emociones de diverso tipo" (Lindón, 2009 , p. 12). Las prácticas nunca son aisladas, sino que responden a un entretejido de secuencias prácticas que se orientan a hacer algo o a cumplir un fin (Ares, 2011).

El análisis de estas prácticas, tal y como fue plantado, sería demasiado imparcial si no se tiene en cuenta el sujeto territorializado, con la carga emocional que posea, dando como resultado una construcción socioespacial de la ciudad. Hiernaux (2007) plantea su estudio como experiencia, desde una postura subjetiva que permita articular los "imaginarios urbanos", con los espacios vividos (Bailly, 1979). La articulación entre ambas dimensiones, permite observar el mundo desde un equilibrio entre una sumatoria de lo material y lo no material, desde la experiencia espacial del sujeto (Lindón, 2008).

Por su parte, el espacio de vida es el espacio frecuentado por cada individuo en base a una serie de nodos mediante los cuales se construye el "espacio concreto de lo cotidiano" (Lindón, 2007, p.7), el hogar, el trabajo, los espacios de ocio. Mientras que el espacio vivido es un concepto más abstracto que refiere a todas las cargas emotivas que el sujeto posee del lugar, las imágenes y los conceptos individuales que le llevan a forjar su propia percepción, y contribuyen a conferirle sentido (Ares, 2011).

Concebiremos al espacio como reflejo de la sociedad, la cual se encuentra ansiosa por ocuparlos y generar territorios de libertad. Sin embargo, esto son territorios de discriminación por género y sexo, y es aquí donde la Geografía debe ingresar en los debates para dar cuenta de las prácticas y conductas socio espaciales; el acceso no es homogéneo y esto es visible en prácticas territoriales diferenciadas (Torres-Rodríguez, 2012). "Pero no podemos olvidar que toda actividad humana se desarrolla en y con el espacio geográfico, del que nos aprovechamos, sobre el cual incidimos, pero que a su vez también nos impone condicionantes" (Sánchez, 1990, p. 191). 
Al interior de los procesos políticos y sociales, las poblaciones se encuentran en constante movimiento y circulación. A esto lo denominaremos movilidad territorial, la cual se entiende: "como el conjunto de desplazamientos en el espacio físico, de individuos o grupos, sea cual sea la distancia recorrida y la duración [...]. Dentro de este complejo conjunto se ubica la movilidad habitual o cotidiana" (Ares, 2012, p. 270). Esta movilidad habitual (Courgeau, 1990; Gutiérrez, 2012), es concebida como la movilidad que posee la población en su día a día dentro del espacio de vida, refiriendo a la realización de actividades (empleo, recreación, atención médica, etc.) y los desplazamientos necesarios para llevarlas a cabo. El análisis inverso, sobre cuáles son las movilidades cotidianas de las personas, nos llevará a reconstruir sus espacios de vida y el entrelazado de sentidos y emociones que estos poseen (Bailly, 1989; Ares, 2011). Culminando con una construcción de "significados personales" originados en los valores culturales propios de esa sociedad.

Para referir al acceso a la ciudad, Harvey (2012) estructura a la sociedad desde dos perspectivas contrapuestas. Por un lado, encontraremos un grupo privilegiado que posee acceso a los servicios, que tiene posibilidades de estudio y de recreación, acceso a trabajos dignos, asfalto, salud, etc. y, por el otro, un grupo marginado y precarizado desde todos los ámbitos públicos y privados, que no tiene las mismas posibilidades de acceso y para los cuales la vida se transforma en una odisea constante para poder poseer un hogar o satisfacer sus necesidades básicas diarias.

El acceso a la ciudad tiene una exclusividad para aquellos que dispongan de una acumulación de capital suficiente que les permita acceder, mantener sus servicios e insertarse en su modelo económico y social. El espacio urbano posee una profunda relación entre la población y el género, siendo este un contexto cargado de interrelaciones por descubrir y de espacios por explorar; por lo tanto, se debe estudiar desde las múltiples expresiones de los ciudadanos en el espacio heterogéneo, donde la diversidad es lo que prima (Torres Rodríguez, 2012).

Aquí el concepto de plusvalía -no refiriendo a la categoría de Marx como parte del sistema de producción capitalista sino refiriendo a la valorización que sufren diversos inmuebles en función de acciones que son externas a los propietarios y que fundamentalmente son llevadas a cabo por el Estado- transforma al espacio en perverso, en un lugar de especulación y de comercio, donde las poblaciones son desplazadas y otras se apropian de lugares dentro de la ciudad; fenómenos que son sucesos a nivel mundial, y del cual Mar del Plata no está exenta (Canestraro et al. 2015).

De esta forma, las ciudades dejan de ser patrimonio común de los habitantes que viven en ellas. Hoy más que nunca en la historia, el acceso a los servicios que las urbes brindan, y la posibilidad de tomar decisiones, es diferenciada (Harvey, 2012). Esto define 
experiencias de vida cotidiana complejas, solidarias y a la vez externas a la voluntad de Ixs sujetxs. El derecho a la ciudad no es sólo el acceso individual o grupal a los recursos de ella, sino que también es el derecho a cambiar y reinventarse de forma colectiva, buscando la diversidad existente en diferentes escalas y debe ser concebida como propuesta de una ciudad mejor (Harvey, 2008; 2012).

Del total de población que compone la ciudad, las dinámicas de género no serán iguales, y la forma de vivir y de circular por esta será condicionada por las características físicas, al mismo tiempo que por el capital que la persona posea para desarrollarse; será un contexto cargado de interrelaciones por descubrir y de espacios por explorar (Torres Rodríguez, 2012). Los espacios son condicionantes del género y opresores de disidencias, sobre todo en ciudades pequeñas, o en las que todavía rigen patrones sociales tradicionales, llevando a las personas a auto-discriminarse en la vida diaria, o bien a restringir sus espacios y actividades para preservarse (Butierrez, 2019).

Partimos del concepto de "identidad" entendido como un sistema de regulación y control de las subjetividades inscriptas en la corporalidad de la/os sujeta/os y en sus externalidades, constituidas históricamente a partir de relaciones de dominio y subordinación diferenciadas, jerarquizadas, artificiales, estables y predeterminadas (Ochoa, 2017, p. 2).

Dentro de estas identidades, surge lo "trans" como un término paraguas de reciente aparición, que busca, de alguna forma, aglomerar identidades disidentes, asociado a la construcción de la identidad de género de las personas, o bien a cómo ellas se perciben, cómo quieren ser identificadas y con qué género quieren ser tratadas. El término engloba a todo un grupo de personas que se encuentre transitando dentro de su identidad de género (Torres Rodríguez, 2012), entendiendo al "tránsito" como un suceso que se produce, de una u otra forma, en todas las personas y que se va manifestando de diferentes maneras.

En el devenir entre el género masculino y femenino surgen identidades que buscan otra forma de presentarse e identificarse, como por ejemplo las personas no binarias. Identidades que deciden no representar estereotipos binarios en su nueva construcción de género (Ochoa, 2017). Por otro lado, "La identidad de género hace referencia a la construcción cultural combinada con el querer ser de cada quien, respecto de la representación de la feminidad, masculinidad o género intermedio elegido" (Espinoza 2008, en Torres Rodríguez, 2011, p.11).

\section{Materiales y métodos}

Para este trabajo se tomó el camino de construir información primaria mediante la propia voz de Ixs sujetxs, por medio de dos momentos. Inicialmente, con una metodología 
cualitativa, con el objetivo concreto de realizar un primer acercamiento a las realidades y problemáticas de la población. En base a esa experiencia inicial, se desarrolló luego una etapa de naturaleza cuantitativa, por permitir una mejor sistematización de los materiales e instrumentos.

En un primer momento, se lanzó una convocatoria abierta y no excluyente hacia personas trans que quisieran colaborar a través de redes sociales. Esto permitió comenzar a reconocer la temática que se estaba intentando abordar, realizando conversaciones con contenido que permitieran indagar sobre algunas nociones generales de la población y conocer mejor el campo en el que se deseaba incursionar.

Esta convocatoria abierta fue lanzada en redes sociales a partir de la aplicación Instagram mediante una publicación temporal, que permitió una rápida difusión. Generando un efecto "bola de nieve" o enlace en red (Scribano, 2008), comenzó a ser replicada por diferentes personas, lo que llevó en pocas horas a que cuatro personas se comunicaran para participar.

Una vez localizadas las personas participantes, por la misma red social se les dieron cuatro tópicos a abordar mediante un discurso libre: (1) cuál es su autopercepción de género, (2) aportar una breve descripción de la zona que habita, (3) indicar lugares que frecuente a lo largo de las semanas; y, por último, (4) señalar lugares que, a su criterio, son cómodos para su habitabilidad y circulación, en contraposición a lugares incómodos que prefiera evitar o que le han generado problemas por su performatividad.

Para esta actividad se aplicaron las técnicas de observación y participación (Scribano, 2008 , p. 34). Estas técnicas implican un proceso que genera "una inclusión consciente y planificada, hasta donde lo permiten las circunstancias, en la cotidianeidad de los grupos de estudio" (Marshall y Rosman, en Scribano, 2008, p. 34) para las primeras etapas del proceso de investigación cualitativa. Dicho proceso permite la inserción al campo mediante la percepción de Ixs individuos, Ixs cuales se transforman en sujetos que nos guían por sus saberes y su vida cotidiana. Si bien esta estrategia fue útil para comenzar a reconocer problemáticas y dinámicas, se creyó necesario una segunda instancia que permitiera relevar más realidades y así profundizar en el estudio.

La conflictividad para el desarrollo de más entrevistas, debido a los recursos disponibles y la coyuntura de Aislamiento Social Preventivo y Obligatorio (ASPO) decretada en Argentina en el marco de la propagación del virus SARS-CoV-2, determinó que en un segundo momento se recabara una muestra cuantitativa. Buscando un relevamiento que permita reconstruir otras realidades y tomando como referencia esta primera entrada a campo, se desarrolló un cuestionario "autoadministrado" y "semi estructurado" (Marradi, et al. 2007, p. 212). La idea de esta estrategia técnica, lejos de ser reduccionista o esencialista, 
buscaba recuperar la experiencia de las personas (y con ellas la de la comunidad trans) en base a diferentes ejes y tratando de aprovechar la virtualidad.

Así, en línea con lo que plantean Sautu et al. (2005), se optó por reconstruir una perspectiva "microsocial" sobre la realidad del universo con el que nos propusimos trabajar, que abarcó la "experiencia individual y la interacción social, que son las fuentes de creación de significados y de bases para la acción concertada y creación y recreación del orden social" (Sautu, et al. 2005, p. 55).

Se decidió, por tanto, construir un instrumento de tipo no probabilístico, conformado por cuestionarios semiestructurados de Google Forms que permitió su fácil difusión por redes sociales mediante un link. El formulario también fue distribuido entre diferentes organizaciones del Estado, ONGs, y lugares reconocidos por las primeras conversaciones con contenido, entendiendo que estos se constituían como puntos de referencia para la población trans en la ciudad. Además, cada persona que participaba era invitada a difundir información sobre el relevamiento contribuyendo en la conformación de la muestra mediante una estrategia de "bola de nieve" / Enlace en red (Scribano, 2008).

El cuestionario se compuso de cuatro apartados, el primero recababa información básica de la persona (edad, percepción de género, nivel de instrucción, lugar de procedencia, etc.). Seguidamente, se relevaba información sobre la situación de empleo de la persona. En un tercer apartado se trabajó con el espacio de vida y el espacio vivido, nociones de movilidad cotidiana, lugares topofílicos, topofóbicos y lugares de miedo en relación con su género/sexualidad, y un último apartado se destinó al acceso a la ciudad.

El instrumento (exceptuando el último apartado) se construyó con un estilo de múltiple respuesta porque, según las experiencias de Marradi et al. (2007), los formularios autoadministrado y en soporte virtual no reciben una buena acogida por parte de los usuarios cuando solicitan respuestas de desarrollo. No obstante esto, se incluyó en cada instrumento una breve descripción del propósito de cada inciso, junto con una "categoría residual" ("otra/o") en las preguntas (tal y como propone Scribano, 2008), para salvar casos de potencial incomprensión o indisposición a responder.

En un último apartado, se trabajó el acceso a la ciudad para comprender las diferencias que el género crea en el espacio urbano. Se decidió aplicar una Escala Likert, que: "son instrumentos psicométricos donde el encuestado debe indicar su acuerdo o desacuerdo sobre una afirmación, ítem o reactivo, lo que se realiza a través de una escala ordenada y unidimensional" (Matas, 2018, p. 39) basada en la escala Nunca / A veces / Habitualmente / Casi siempre / Siempre. Este tipo de escala, según Matas (2018) se recomienda para las investigaciones sociales. De esta forma, se propusieron 12 afirmaciones 
de situaciones, y se debía seleccionar qué tan habitual o poco habitual era que eso les sucediera a lo largo de la semana.

Se debe reparar en que la Escala Likert posee ciertos sesgos a tener en cuenta (Matas, 2018): el primero vinculado con un cierto estilo de respuesta (el cual puede tender a ser extrema, mantenerse siempre en rangos intermedios o tender hacia lo socialmente deseable). Y el segundo, que la respuesta intermedia suele tender a representar ambivalencias de la persona sobre el tópico sobre el cual se le interpela.

Tanto para la presentación de la zona de estudio, como para referenciar la localización de las respuestas a la encuesta, se optó por la utilización de Sistemas de Información Geográfica de software libre y código abierto, particularmente Qgis Madeira, para simplificar el proceso de sistematización y referenciación de los datos.

\section{Resultados}

La presentación del análisis de la información que se recabó se va a dividr en dos momentos. Primero se presentarán los datos cualitativos, relevados con conversaciones con contenido, y luego los datos cuantitativos, relevados mediante cuestionarios. Ambos serán presentados de forma explícita y absoluta, ya que se priorizaron las propias voces de las personas narrantes por sobre las interpretaciones de quien investiga. Los análisis serán generales, de manera que se pueda preservar las identidades personales, intentado visibilizar las realidades sin exponer ni (re)victimizar a Ixs participantes.

Se comenzará reconstruyendo las primeras narrativas obtenidas a partir de las conversaciones con contenido. Estas serán analizadas conforme los tres tópicos que se les fijó a cada uno de los cuatro participantes. Cuando se refiera a fragmentos textuales, se presentará la correspondiente transcripción sintética. Vale recuerda que estos textos fueron recabados por redes sociales, mediante una sumatoria de mensajes de texto y, en muchos casos, mediante mensajes de voz, por lo que, si bien fueron transcriptas textualmente, se agregó aquí mayor fluidez a la redacción con algunas acotaciones en paréntesis para su mejor comprensión. Se omitieron las referencias a los lugares físicos para poder garantizar el anonimato de cada sujetx.

Primero se trabajó con la autopercepción de género. Tres personas se percibieron mujeres y una se percibió varón:

Yo percibo mi género como que soy una mujer, pero una mujer que no está completa, porque me falta a mi más hormonas, me faltan operaciones, estoy en el trámite de todo eso para llegar a ser completamente mujer, pero tampoco soy hombre, es bastante difícil (S: 18 años). 
Luego se les pidió que dieran una breve descripción de la zona que habita y los lugares que frecuentan a lo largo de las semanas. Esto fue con el objetivo de empezar a reconocer sus espacios de vida y comprender cuál es su movilidad cotidiana,

Mi vida se basa prácticamente en cursar y trabajar, durante la semana curso mucho en [...], y durante el finde me dedico a trabajar [...]. Algunos días nos reunimos con el grupo de políticas de género y eso me predispone a averiguar en diferentes lugares información que me pueda ser útil o que me junto para estudiar. Yo milito en diferentes organizaciones para buscar la inclusión de género [...] (F: 24 años).

[...] trabajo [...] y mi día a día son así, vivo sola, dedico mi día a trabajar, miro series y visito a mi familia y amigos [...] No Salgo a bailar porque no me opere, estoy gorda y eso... y bueno no me siento cómoda como para salir, no me gustan los boliches gays por la gente que concurre y los boliches como Quba y Mr Jones no soy aceptada (L: 20 años).

[...] en mi vida diaria principalmente estudio Programación [...], por las mañanas hago pasantías y a la tarde curso en la facultad, y suelo estar mucho por el centro en general. No me suelo alejar mucho del centro salvo cuando voy al puerto a cursar (J: 19 años).

[...] yo no voy a la escuela porque me hacen mucho bullying, así que dedico mis días a hacer módulos de la escuela, encerrada en mi casa, normalmente no salgo en todo el día ya que también trabajó acá (refiriendo a su casa) [...], fue muy complejo mi paso por la escuela, me trataban muy mal, me agredía psicológicamente y hasta llegó a ser físico [...]. Me la paso encerrada en mi casa, no salgo a ningún lado a menos algún café a alguna amiga o un boliche [...] pero no me gusta salir. A dónde si salgo todos los sábados a la tarde $u$ otros días de la semana, es la casa de mi tía. Suelo ir eventualmente a (parque céntrico), pero no tanto como antes [...] también voy al centro muy de vez en cuando, pero suelo estar con mi familia casi todo el tiempo. [...] (J: 19 años).

Por último, se les pidió que refieran a lugares que, según su criterio, son cómodos para su habitabilidad y circulación, en contraposición a lugares que prefieren evitar o que les genera problemas por su género. Esto dejó al descubierto distintos miedos e inseguridades, llevando a localizar y referenciar situaciones y zonas de la ciudad que les generaban rechazo, y otras que, por el contrario, eran habituales y elegidas por ellxs:

Los lugares que a mi me encantan son los relacionados a la universidad, ya que es ese lugar que te acerca al lugar que encuentro entre la teoría y la realidad, me encanta pasar el tiempo en la universidad, me siento muy acompañada y disfruto de las discusiones y debates políticos y teóricos que se puedan llegar a dar. Los lugares que no frecuento son aquellos espacios en los que no comparto políticamente o jurídicamente, porque yo siempre voy a partir de la base de que todos somos personas y todos somos sujetos de derecho. Muchas veces una se cruza con gente que es radfem (feminismo extremo radical) o terfs (subgrupo de feministas que están ampliamente en contra de las identidades, experiencias y derechos de las personas de géneros 
disidentes) [...]. Porque si los lugares no me gustan y no me siento cómoda, trato de evitarlos por mi propio bien (F:24 años).

Me movilizo mucho por bares [...], sobre todo en remis, ya que tomar colectivo para mi conlleva una visión constante de parte de las personas y además no me gusta el contacto, igual los tomo de vez en cuando, pero no son de mi agrado. Lo mismo me sucede con el centro de la ciudad, el cual prefiero evitar y sobre todo la peatonal, no sólo por la gente, por las miradas, sino que al contrario sos el punto fijo de todos, nada que ver con Buenos Aires (ciudad) (L:20 años).

[...] trato de no frecuentar lugares con poca circulación de gente por una cuestión de seguridad, porque si bien soy un chico trans muchas veces la gente me sigue identificando como mujer. Me han robado varias veces así que trato de tener cuidado. Vivo cerca de (parque céntrico y tradicional de la ciudad) y por eso suelo manejarme caminando o en colectivo (J: 19 años).

Esta dinámica permitió conocer algunas realidades de las personas trans entrevistadas. Se reconocieron problemas con los espacios educativos, además presentaron mayor énfasis en la violencia simbólica o verbal que se puede vivir muchas veces en las calles y en los espacios de ocio de la ciudad. Por último, se focalizó en los espacios de inseguridad o miedo. La zona céntrica fue nombrada en las charlas como un espacio de riesgo o de miedo en ciertas situaciones, ella también es una zona muy visitada o transitada por Ixs sujetxs, lo cual transforma sus actividades diarias en actividades de riesgo, por las cuales teme sufrir algún tipo de violencia.

En un segundo momento, fueron aplicados los cuestionarios. Se distribuyen desde el 22 de mayo del 2020 al 22 de junio de 2020, reuniendo un total de 20 respuestas localizadas en 15 barrios de la ciudad de Mar del Plata. Como podemos observar en la Figura 2, la mayoría de las respuestas se agrupan en la zona céntrica, y en segundo lugar en la zona costera sur.

Dado que en esa época la ciudad se encontraba confinada debido a la pandemia por el virus SARS-CoV-2, al principio del relevamiento (y en ítems específicos) se solicitó responder en base a una construcción del cotidiano que exceda la coyuntura, de manera que se pueda paliar esa interrupción -coyuntural- de las actividades de la mayoría de la población. 


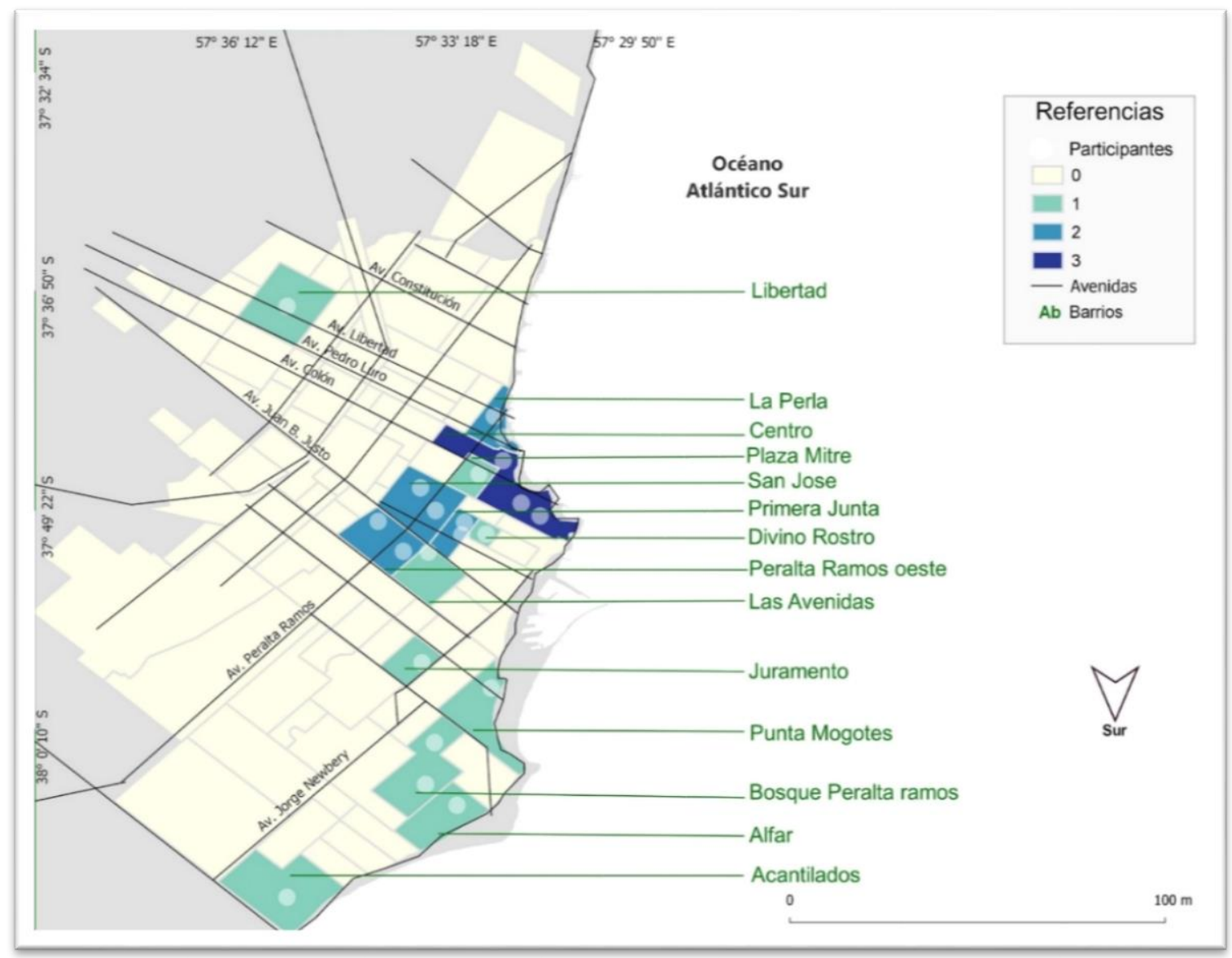

Figura 2: Nivel de participación y barrios de les encuestades 3

Fuente: Elaboración personal con base en datos obtenidos en 2020, mediante el empleo del SIG de acceso libre Qgis.

El conjunto de encuestados corresponde a un rango etario comprendido entre los 16 y los 34 años de edad, con lugar de nacimiento en "Mar del Plata" (17 personas) u "otras localidades de la provincia de Buenos Aires" (3 personas).

Comenzando con el análisis de los resultados, se permitió que cada persona pudiera elegir los géneros que (mejor) construyeran su identidad. $Y$ esto porque se considera relevante poder visibilizar la autopercepción de cada participante ya que, tal y como plantea Torres Rodríguez (2012), el identificarse con un género es una batalla que la persona ha llevado consigo misma y luego con la aceptación social. En general, las personas que participaron se encuentran cómodas con la definición "trans", al igual que "chica trans" o "chico trans". Pero también hubo un grupo que no se sintió representado ni por chica, ni por 
chico, y que optó por percibirse como: "trans no binarie" o simplemente "no binarie". Este último caso agrupó a siete participantes.

El nivel de instrucción fue muy variado dentro de la población analizada. La mayoría indicó nivel de instrucción secundario completo e incursionó en niveles de estudio superior no universitario o universitario, los cuales a veces han tenido que abandonar por la presión social o por discriminación, esto según fue descrito:

[...] hay muchas ocasiones en que dejo de hacer algo o intento cambiar mi performatividad de género para no sentirme vulnerable, pero circulo de todas formas. Es igualmente opresivo, porque no estoy tranquilo. No sé si pude expresar eso. [...], como dije, la UNMDP me expulsó por trans, fue re violento todo el año pasado en plena transición (Trans No Binarie, 27 años).

Si esto se relaciona con que 15 de les 20 participantes manifestaron que habían tenido problemas en el lugar donde estudian o estudiaron, por lo menos alguna vez y debido a su aspecto o debido a expresión de género que eligieron, entonces, estamos hablando de un ambiente que se transforma en hostil para las disidencias.

En relación al empleo, del total de participantes, diez manifestaron trabajar durante la última semana (cuatro se encontraban en cuarentena, vacaciones o licencia; mientras que seis efectivamente lo realizaron), tres se encontraban buscando empleo y diez no trabajaron ni buscaron empleo.

En cuanto a las personas que poseen un empleo (independientemente de que lo hayan podido ejercer con normalidad virual-remota o no), pudimos reconocer una variedad de profesiones y lugares. La mayoría están relacionadas con atención y venta al público, estética y salud. Se realizaban mayoritariamente en un "local/ oficina/ industria/ taller", a excepción de quien manifestó realizar trabajo sexual, ejercido en el hogar de los clientes y, por otro lado, el "malabarismo o emprendedorx" que se realiza principalmente en el espacio público.

En cuanto a las personas que buscan empleo, se notó que ellas no logran ejercer la profesión para la que se han formado, situación que relacionan con el género adoptado. La incorporación laboral a espacios profesionales se manifestó como una gran problemática, aseguran tener serias para acceder a un empleo y en algunos casos, incluso, deben "invisibilizar" su opción de genero (vestirse con ropa que no les represente).

Desde la percepción económica, se dejó en evidencia que mayoritariamente los ingresos que perciben por sus empleos no alcanzan a cubrir las necesidades básicas. En suma, la mayoría de Ixs participantes aseguraron que conseguir empleo, o directamente acceder al ámbito laboral, es un problema habitual para las personas trans. 
Comenzando a reconstruir los espacios de vida de Ixs entrevistadxs, se pudo encontrar un fenómeno de topofilia por la ciudad, así como también por las formas de habitar. Cuando se preguntó ¿Por qué vivían en Mar del Plata, se respondió:

Tiene su parte mágica y queer. Mi manada de amigues esta acá. Pero he pensado en mudarme porque lo facho a veces es mucho (Trans No Binarie, 34).

Es una ciudad grande pero no demasiado como para asfixiarme (Trans No Binarie, 23).

Porque amo el mar (Chica trans, 24).

No decido, ya vivo acá, pero si es porque no me voy, es por mi familia, conocidos y principalmente por la costa, donde me gusta ir a patinar (Chica trans, 26).

En línea con esto, también se manifestó aprecio por el hogar que habitan, el cual manifestaron, es casa o departamento (excepto una persona que manifestó vivir en un rancho). Pero no dejaron de surgir situaciones de opresión con relación al hogar,

Me parece importante tener en cuenta que muchas personas trans viven con más personas que no aceptan, respetan o entienden su identidad de género. Esto nos genera mucha angustia y para quienes no podemos mudarnos ansiedad de quedar en la calle o que nos echen de nuestra casa (No Binarie, 22).

En cuanto al acceso a los sistemas de salud, todos los sujetxs encuestadxs aseguraron, en gran medida, tener acceso a los sistemas de salud. La mayoría accede a ellos mediante el sistema público (en hospitales y centros de atención primaria de la salud) o el sistema privado (en hospitales, clínicas y consultorios); sólo un caso afirmó utilizar medicinas alternativas, y otro manifestó no acceder al sistema de salud. Sin embargo, esto no eximió que comentaran problemas o falta de confianza acerca los sistemas de prestación médica:

Sólo hay un médico al que voy que es lo más, el resto de especialistas los evito (Trans No Binarie, 27 años).

La movilidad cotidiana por la ciudad la suelen realizar caminando, en bicicleta o transporte público de colectivos. Pocos tienen acceso a transporte propio, pero se evidencia una relación directa con la ausencia de opciones de transporte y el mal estado del asfalto, principalmente en la zona Sur de la ciudad. 
En cuanto a los lugares de frecuentación cotidiana, se propusieron diferentes espacios, de los cuales cada participante seleccionó los que eran habituales a lo largo de la semana. De estos diferentes espacios, algunos fueron extraídos de las primeras conversaciones con contenido y otros fueron incorporados para completar un abanico diverso de posibilidades. Inicialmente, se les solicitó que seleccionen cuáles eran los lugares que consideraban de frecuentación cotidiana. Como resultado, podemos decir que se notó una predominancia de los espacios verdes, las casas de amigos y los espacios de estudio (Figura $3)$.

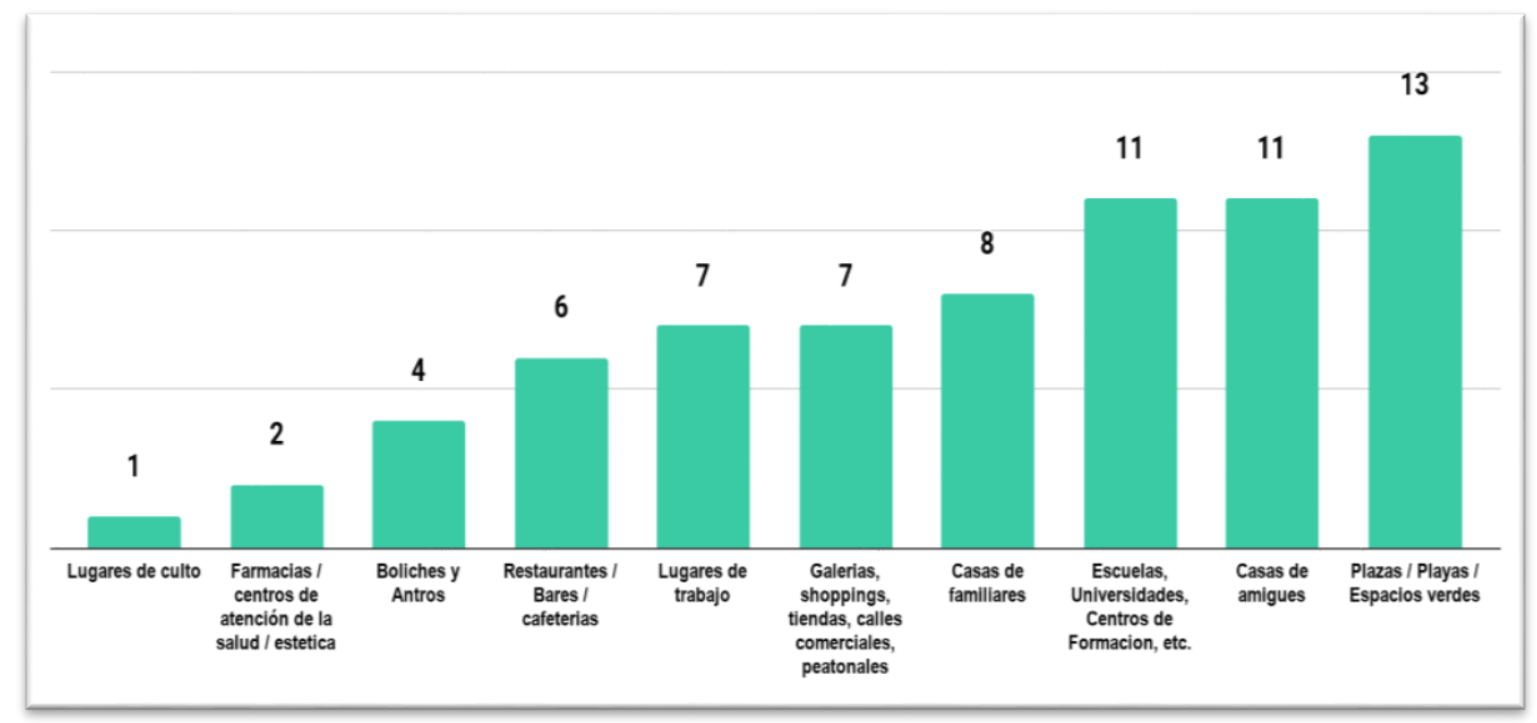

Figura 3: Lugares de frecuentación cotidiana de Ixs encuestadxs

Fuente: Elaboración personal con base en datos obtenidos en 2020.

Luego, se buscó trabajar con los denominados por Lindón (2009) como espacios de miedo, de poca frecuentación o "topofóbicos" (Tuan, 2007). Entonces, se les pidió a Ixs encuestadxs que seleccionen todos los lugares que, a su parecer, restringían o limitaban las posibilidades de desarrolla su vida diaria en la ciudad. Se propusieron los mismos espacios que en la consigna previa, pero incorporando las calles de la ciudad y la zona céntrica, ya que en las conversaciones con contenido habían surgido como lugares "conflictivos". Así entonces, 15 personas evidenciaron haber sufrido diferentes situaciones de violencia en estos lugares (Figura 4). 


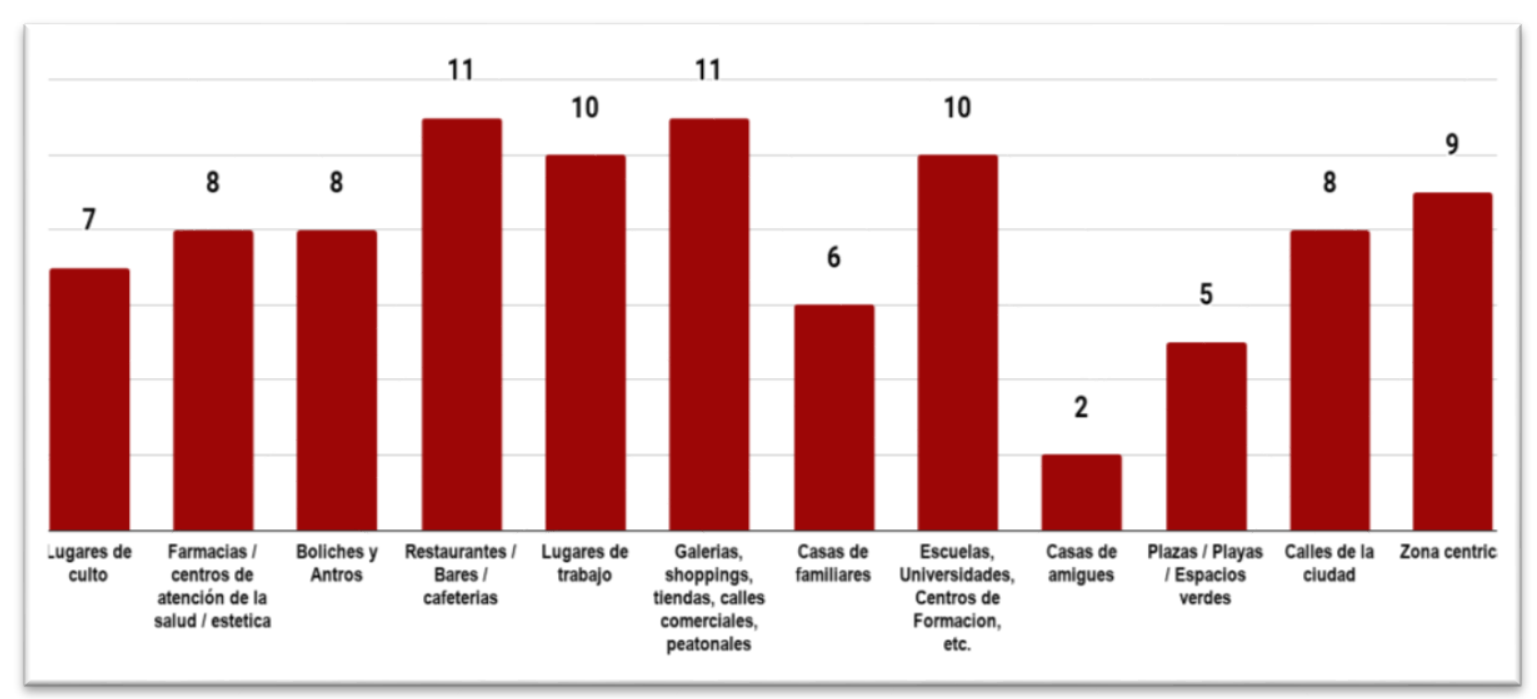

Figura 4: Lugares que restringen o limitan la posibilidad de desarrollarse

Fuente: Elaboración personal con base en datos obtenidos en 2020.

Como podemos ver, una gran cantidad de los sujetxs consultados sufrieron o perciben de forma violeta los espacios públicos de la ciudad, principalmente los comerciales (bares, restaurantes, cafeterías, boliches, farmacias, etc.) y los espacios públicos urbanos (zona céntrica, calles de la ciudad, etc.). También resultó llamativo cómo se han manifestado con respecto a los lugares de trabajo y a las casas de estudio, aparentemente estos lugares de la ciudad les generaron múltiples problemas y conflictos, en línea con las conversaciones con contenido.

Para trabajar el acceso que las personas poseen a la ciudad, se aplicó la Escala Likert (Matas, 2018). Se propusieron doce afirmaciones de situaciones que podrían sucederle a la persona. Estas se seleccionaron mediante las conversaciones con contenido realizadas previamente y con base también en las indagaciones realizadas por Torres Rodríguez (2012) en sus entrevistas de acceso a la ciudad para comprender las diferencias que el género crea en el espacio urbano. 


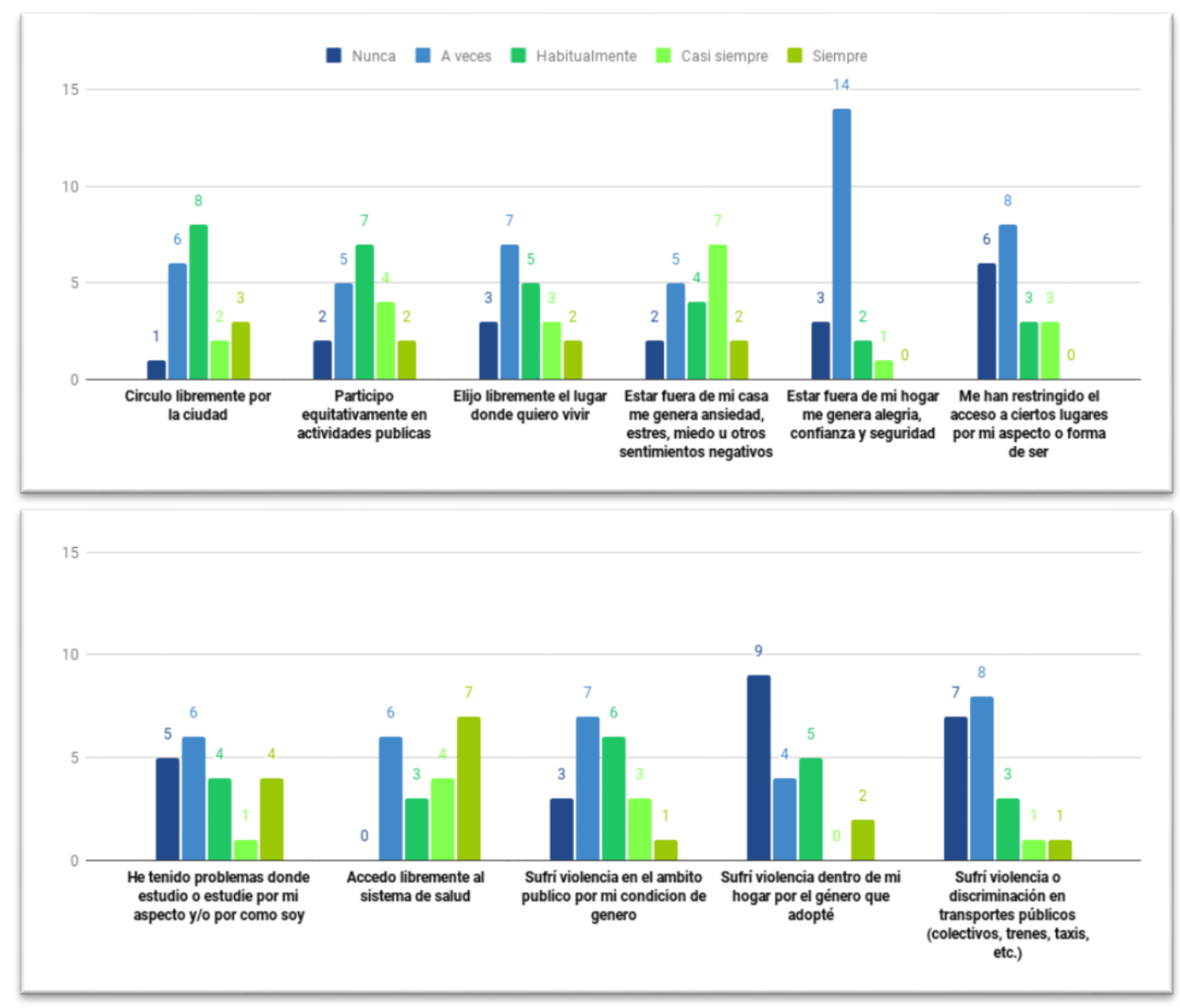

Figura 5: Acceso a diferentes espacios para Ixs encuestadxs

Fuente: Elaboración personal con base en datos obtenidos en 2020.

En la Figura 5 se presentan los resultados. Se debe recordar que existe un sesgo de estilo en la respuesta intermedia, referido a cierta tendencia por elegir las alternativas moderadas, evitando las extremas (Matas, 2018, p. 44). Es importante tener esto en cuenta para interpretar mejor los argumentos que cada participante ha tenido, buscando reproducir la respuesta socialmente deseable.

Como se observa, los resultados representan una gran dificultad para la circulación libre de los sujetxs por la ciudad, para realizar actividades, para acceder libremente a la vivienda, para ingresar a los espacios públicos en general, a los de estudio y a los vinculados con los sistemas de salud en particular; y para utilizar transportes urbanos. Son pocas las oportunidades en las que se seleccionaron las opciones: "siempre" o "casi siempre". 
Por lo visto, el acceso a la ciudad no es igual para todas las personas, los valores más críticos se dieron en la zona más lejana del centro. Las emociones, trabajadas por Lindón (2009, p. 6): “como ventana para comprender la construcción social de la ciudad", han dejado en evidencia inseguridades, miedos y ansiedades al momento estar fuera del hogar. Las elecciones sobre dónde vivir, qué transporte utilizar, a qué sistema de salud acceder o dónde y cuándo estudiar, se presentaron como situaciones complejas e irregulares, en muchos casos limitadas -o anuladas- por la elección de género de las personas consultadas.

Finalmente, como observamos en la Figura 5, las manifestaciones de violencia no solo ocurren en ámbito público (en el transporte, en los espacios de salud o de estudio), sino que resultan más preocupantes porque ocurren también dentro del hogar. Estas situaciones, en suma, complejizan el acceso a la ciudad, es decir, el acceso individual o grupal a los recursos de la urbanización; al mismo tiempo que limitan el derecho a cambiar y reinventarse de forma colectiva en la ciudad (Harvey, 2008).

No se puede dejar de remarcar la sororidad que se puede percibir dentro de las personas que han participado. En este sentido, Wayar (2019), sostiene que solo la unidad como sociedad logrará cambiar las pequeñas realidades para llegar a construir una "nostredad". Eso queda plasmado en las inquietudes de Ixs participantes:

Yo soy un caso excepcional ya que ser trans es muy caro, por las hormonas, las operaciones, la ropa, los maquillajes. Si no tienes un trabajo fijo, o algún familiar que te banque, es casi imposible, y ese es el problema del 95\% (fuente no encontrada) de la población trans que termina en las calles porque no encuentra otra salida (Chica Trans, 20 años).

[...] yo soy una persona con muchos privilegios al lado de otras personas trans (Trans No Binarie, 22).

[...] al llevar una vida súper distinta a la mayoría de las trans hace que cuando alguien me pregunta o pienso en la vida de todas las personas trans que son como yo y no tienen las mismas posibilidades que yo por el simple hecho de ser quienes son y siempre quisieron ser me pone MUY mal, me hace pensar en que tan triste es el mundo y cerrado en el que una persona no puede ser libre sin que haya una sociedad que la castigue por simplemente querer ser feliz y me encanta que cada vez se nos quiera incluir y visibilizar más (Chica trans, 20).

Personalmente creo que, si bien sufrí poco y nada de discriminación por mi condición, tuve la suerte de encajar dentro de la figura estética hegemonía. No es el caso de amigas que sufren constantemente la discriminación por sus rasgos "masculinos". Por eso creo que es importante mantenerse unidas y no competir por quien "pasa" desapercibida, palabra que odio. No importa si tenes o no operaciones o si tomas o no hormonas, todas somos parte del mismo colectivo (Chica trans, 26). 
Si bien cada dinámica es particular, y cada usuarix habita y recorre la ciudad de forma diferente, los resultados de los cuestionarios que se pudieron recabar han evidenciado situaciones específicas que se manifiestan en la ciudad. Desde ya que no es posible generalizar, pero sí se puede mencionar que se han rescatado situaciones de violencia, espacios topofílicos y topofóbicos, conflictos, en definitiva, para las identidades disidentes que aún no se han salvado en el devenir social.

\section{Reflexiones finales}

En este trabajo se buscó indagar sobre la dinámica urbana de la población trans en Mar del Plata, reconociendo sus espacios de vida con el propósito de analizar cómo se dan sus posibilidades de acceso a la ciudad. La ausencia de investigaciones similares dentro del territorio de análisis ha llevado a complejizar la posibilidad de comparar y revisar la información obtenida. Por eso, esta primera indagación ha tenido la intención de permitir un acercamiento inicial sobre la situación particular de dicha ciudad costera, sin aspirar a ser reduccionista ni generalista. Sin embargo, reconocemos grandes similitudes con los hallazgos regionales de Dairouiche (2019), Torres-Rodríguez (2012) o Fuentealba-Matus (2016).

Mediante el empleo de las técnicas aplicadas, hemos podido reconstruir territorios cotidianos de diferentes personas trans que viven en la ciudad. Si bien las realidades han sido muy diversas, podemos señalar que el acceso a la ciudad se encuentra discriminado por un fuerte componente de clase y género. Las realidades contrapuestas y la coartación de derechos, se manifiestan a flor de piel; dando como resultado habitabilidades parciales y discriminadas, sensaciones de miedo, y hasta tendencias al aislamiento... lo que parece transformar la dinámica urbana.

La población trans se encuentra relegada en la ciudad. Falta mucho para poder alcanzar un acceso real a los lugares, al travajo ${ }^{4}$, al estudio y a la salud. Los espacios tanto públicos como privados, pueden transformarse en verdaderas barreras limitantes para la libre expresión de género que cada persona adopta, y para el desarrollo de su propia vida. Se debe comenzar a trabajar en lograr espacios igualitarios e incluyentes, que le permitan a cada performatividad expresarse libremente, ya que el mundo es dominio de todxs.

\footnotetext{
4 "Se escribe con ' $v$ ' para visibilizar la difícil realidad con la que choca cualquier persona trans, travesti o transexual cuando se dispone a buscar empleo y se encuentra con múltiples obstáculos derivados de la discriminación, el prejuicio, el estigma, sumados a una tasa de desempleo general que aumenta. Sirve para que se sepa, para que se hable, para que exista. Lo que no se nombra, no existe" Fragmento de entrevista a legisladora de la Ciudad de Buenos Aires, Ofelia Fernández, realizada el 25/06/2019 (en línea: http://www.infogremiales.com.ar/noticia.php? $n=58803$ consultado el 14/08/2020).
} 
Este primer acercamiento, nos ha permitido, en primer luar, conocer algunas realidades de personas trans-travestis-no binarias que habitan y recorren la ciudad y, en segundo lugar, visibilizar y comprender algunos lineamientos generales del habitar diario de las identidades disidentes en una ciudad intermedia de Argentina. Queda mucho por pensar, reflexionar y por conocer, pero la puerta está abierta para continuar indagando las Geografías Sensibles y de Género en Mar del Plata. Se espera que este tipo de estudio, unido al activismo, sean el motor de cambio para reconstruir las realidades y repensar las problemáticas sociales.

\section{Referencias bibliográficas}

Ares, S. (2011). Espacio de vida cotidiano, espacio vivido y territorio en Chapadmalal, Partido de General Pueyrredon. Maestría en Ciencias Sociales y Humanidades, con mención en Sociología. Universidad Nacional de Quilmes.

Ares, S. (2012). Entre el aislamiento y la libertad. Prácticas de movilidad cotidiana y diferencias de género en el sudeste bonaerense (Argentina). Brazilian Geographical Journal: Geosciences and Humanities, 3(2), 267-298.

Bailly, A. (1979). La percepción del espacio urbano: conceptos, métodos de estudio y su utilización en la investigación urbanística. Instituto de Estudios de Administración Local.

Bailly, A. (1989). Lo imaginario espacial y la geografía. En defensa de la geografía de las representaciones. Anales de geografía de la Universidad Complutense, 9, 11-19.

Barbosa de Souza, M. y Ornat, M. (2019). Segregação Urbana, Gênero e Sexualidades: caminhos de reflexão em tempos de retrocessos. En D. Lan (Ed.), Actas del IV Seminario Latinoamericano de Geografía, Género y Sexualidades (pp. 199-206). Universidad Nacional del Centro de la Provincia de Buenos Aires.

Butierrez, M. (2019). Escribiendo fronteras en Ixs cuerpxs: Una etnografía sobre las prácticas espaciales y políticas de mujeres trans de la provincia de Salta. En D. Lan (Ed.), Actas del IV Seminario Latinoamericano de Geografía, Género y Sexualidades (pp. 247-254). Universidad Nacional del Centro de la Provincia de Buenos Aires.

Canestraro, M., Guardia, C. y Layus, E. (2015). A propósito de la recuperación de plusvalías urbanas: notas sobre intervenciones recientes en el Municipio de General 
Pueyrredon. En P. Lucero (Ed.), Atlas de Mar del Plata y el partido de General Pueyrredon II (pp. 48-50). Eudem.

Courgeau, D. (1990). Nuevos enfoques para medir la movilidad espacial interna de la población. Notas de Población CEPAL, (50), 55-74. http://repositorio.cepal.org/handle/11362/12927

Darouiche, C. (2019). Condiciones de vida, sociabilidad y vínculos de parentesco entre las mujeres trans que realizan sexo comercial en la ciudad Mar del Plata. Tesina de licenciatura en Sociología. UNMdP.

Fuentealba Matus, F. (2016). La visibilidad como opción: Manifestaciones espaciales de lo queer en la ciudad de Santiago. Revista de Geografía Espacios, 6(12), 53-70. https://doi.org/10.25074/07197209.12.661

García Ramon, M. (2008). ¿Espacios asexuados o masculinidades y feminidades espaciales?: hacia una geografía del género. SÉMATA, Ciencias Sociais e Humanidades, 20, 25-51.

Gutiérrez, A. (2012). ¿Qué es la movilidad? Elementos para (re) construir las definiciones básicas del campo del transporte. Revista Bitácora Urbano Territorial, 21(2), 6174.

Harvey, D. (2008). El derecho a la ciudad. Conferencia en el Departamento de Geografía. Universidad de Lund.

Harvey, D. (2012). Ciudades rebeldes. Del derecho de la ciudad a la revolución urbana. Ediciones Akal.

Hiernaux, D. (2007). Los Imaginarios Urbanos: de la teoría y los aterrizajes en los estudios urbanos. EURE: Revista Latinoamericana de Estudios Urbano-Regionales, 33(99), 17-30.

Hiernaux, D. (2008). 'Geografía Objetiva' versus 'Geografía Sensible': Trayectorias divergentes de la Geografía Humana en el siglo XX. Revista ANPEGE, (4), 2945.

Ibarra García, M. y Escamilla Herrera, I. (2016). La geografía feminista, de género y de la sexualidad en México, un saber en crecimiento. En M. Ibarra García e I. Escamilla Herrera (Eds.), Geografías feministas de diversas latitudes (pp. 209238). UNAM, Instituto de Geografía.

Jiménez-Jiménez, J; Hoyos-Martínez, J. y Álvarez-Vallejo, A. (2014). Transporte urbano y movilidad. Quivera, 16(1), 39-53. 
Lan, D. (2016). Los estudios de género en Argentina. En M. Ibarra García e I. Escamilla Herrera (Eds.), Geografías feministas de diversas latitudes (pp. 55-70). UNAM, Instituto de Geografía.

Lan, D. y Veleda da Silva, S. (2007). Estudios de geografía del género en América Latina: un estado de la cuestión a partir de los casos de Brasil y Argentina. Documents d'anàlisi geogràfica, (49), 99-118.

Lindón, A. (2007). El constructivismo geográfico y las aproximaciones cualitativas. Revista geográfica Norte Grande, 37, 5-21.

Lindón, A. (2008). De las geografías constructivistas a las narrativas de vida espaciales como metodologías geográficas cualitativas. Revista ANPEGE, 3, 7-26.

Lindón, A. (2009). La construcción socioespacial de la ciudad: el sujeto cuerpo y el sujeto sentimiento. Revista Latinoamericana de Estudios sobre Cuerpos, emociones y sociedad, (1), 6-20.

Matas, A. (2018). Diseño del formato de escalas tipo Likert: un estado de la cuestión. Revista Electrónica de Investigación Educativa, 20(1), 38-47.

Marradi, A., Archenti, N. y Piovani, J. (2007). Metodología de las ciencias sociales. Emecé editores.

Ochoa, L. (2017). Demandas laborales e identidades no binarias. № Jornadas CINIG de Estudios de Género y Feminismos y III Congreso Internacional de Identidades, pp. 38-59.

Sánchez, J. (1990). Espacio Economía y sociedad. Siglo XXI de España editores.

Sautu, R., Boniolo, P., Dalle, P. y Elbert, R. (2005). Manual de metodología: Construcción del marco teórico, formulación de objetivos y elección de metodología. Consejo Latinoamericano de Ciencias Sociales (CLACSO).

Scribano, A. (2008). El proceso de investigación social cualitativo. Prometeo Libros.

Silva, J., Ornat, M. y Chimin-Junior, A. (2013). Geografías malditas: cuerpos, sexualidades y espacios. Cada Palabra.

Torres Rodríguez, M. (2012). Vivencias de sujetos en procesos transexualizadores y sus relaciones con el espacio urbano de Santiago de Chile. Tesis de Maestría. Brasil: Universidade Estadual Paulista.

Tuan, Y. (2007). Topofilia: Un estudio de las percepciones, actitudes y valores sobre el entorno. Editorial Melusina. 
Vila Nuñez, F. (2018). LGBTIQ+: Vulnerables, disidentes, resistentes. En El atlas de la revolución de las mujeres (pp. 88-91). Le Monde diplomatique, Capital Intelectual.

Wayar, M. (2019). Travesti. Una teoría lo suficientemente buena. Muchas Nueces.

\section{Otros documentos consultados}

Colombara, M. (2017). Geografía con perspectiva de género: Estado del arte en América Latina. Encuentro de Geógrafos de América Latina XVI, La Paz, Universidad Mayor de San Andrés.

Colombara, M. (2019). La geografía de género en argentina: breve panorama. Congreso Nacional de Geografía de Universidades Públicas, 7. La Plata, Universidad Nacional de La Plata.

Instituto Nacional de Estadísticas y Censos - INDEC (octubre, 2020). Proyecciones de la población por partidos. Provincia de Buenos Aires, República Argentina. https://acortar.link/1q3cNj

Larreche, J. I. y Ercolani, P. (2018) Identidad barrial: las sexualidades LGBT como corolarios. El caso de la ciudad intermedia de Bahía Blanca. Jornadas platenses de Geografía. La Plata, Universidad Nacional de La Plata. 